\title{
SIMULATION SERVICE PROVIDING IN A DISTRIBUTED SIMULATION ENVIRONMENT
}

\author{
Alexey Cheptsov \\ Donetsk National Technical University, 83000, Donetsk, vul. Artema 58, \\ e-mail: lex@cs.dgtu.donetsk.ua, www.donntu.edu.ua
}

\begin{abstract}
Simulation of complex dynamic systems is an interdisciplinary problem. To solve this problem the author suggests a distributed simulation environment as a new form of simulation means system organization that provides web-based distributed simulation of engineering and industrial tasks as simulation services using Discrete Event Simulation Framework on the basis of Grid infrastructure. The Unified Process of Simulation Services Development based on Unified Modeling Language is proposed.
\end{abstract}

Keywords: Distributed simulation environment, system organization, simulation service providing.

\section{INTRODUCTION}

Internet as a central communication environment and progress of information technologies for the last 10 years have had a strong influence on the simulation technology and especially on web-based simulation that is an important research direction at the area of Discrete Event Simulation (DES) $[1,2,3]$. At the same time the integration of Application Service Providing into Business-to-Business Communications has given the software developers a functional possibility of Service Providing also for tasks of industrial simulation [4,5].

The classical Application Service Providing is founded on interactions between user and computer (computer application). Development of XML and related technologies has allowed data exchanges between separate software components by means of standard communication protocols and interfaces and acts as a basic point in wide-spreading simulation resources into the internet infrastructure based on Web Services technology, Grid computing and Simulation Service Providing. Such standardization allows functional transforming of the Internet as an Information Grid to a Service Grid, in that information and application resources are being requested both by users and by application (software components) transparently [6].

Organization of simulation as an Application Service Providing (Simulation Service Providing, $S S P$ ) requires new approaches of simulation means system organization. A new generation of simulation tools is being developed no more as simple software tools, but as distributed simulation environments those are an infrastructure for integration of software systems and concentrate the most modern engineering and information-technical software solutions at the area of simulation technology. This requires special developing frameworks based on Service Oriented Architecture (SOA). The clients in such frameworks are connected to the server environment through the standard internet protocols (Fig. 1).

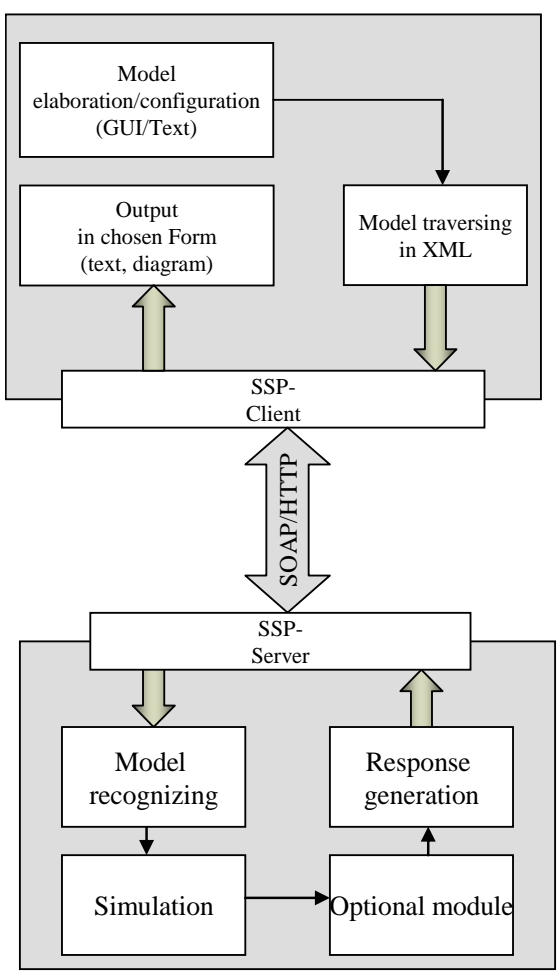

Fig. 1 - Schematic construction of SSP-Proceeding. 
Grid-Computing [7] - a term that means a Computer-, Network- and Software-Infrastructure of resources distribution with the aim of virtual organization tasks solving - allows to develop Simulation Services in distributed simulation environments on a basis of Open Grid Service Architecture (OGSA). OGSA technologies make possible to connect within a single communication and developing framework many SSP-Clients and SSP-Servers as shown in Fig. 1 with further distribution of Grid-Services between ServerResources, what takes use of them to a perspective direction of simulation technology's development and is an important progress in experience accumulation for solving engineering problems.

Herewith the following main requirements are to be taken into account: preparing user-friendly interactive dataset in suitable for engineers graphical and numerical form; integration with existing systems of industrial automation; models correspondence to specifications of problem domain's industrial processes with an understandable and engineers-oriented representation of simulation study; models providing with special training simulators for operators.

The technological potential of Grid infrastructure is imperative for scientific progress. It enables simulation researches and visualization by solving important industrial tasks those would otherwise remain undiscovered and unexplained - e.g. in climate research or material science. Implementation of new approaches of SSP helps researches carry out detailed simulations and are extremely valuable for industry as well because of enabling noticeable reduction in development's and practical use's costs.

\section{OBJECTS AND TASKS OF THE SIMULATION SERVICE PROVIDING. COMPLEX DYNAMIC SYSTEMS.}

The goal of Grid-based SSP is to build a large scale resource sharing infrastructure that enables easy and transparent access of engineers to simulation resources, calculating various simulation sub-tasks on the most suitable computing architecture and subsequently combining the results. It aims also at speeding up scientific simulations for such application areas as for example, weather prediction, astrophysics, bioinformatics, aerodynamics, earth quake research, ground water pollution, multi particle physics and other. All this application areas can be defined by term of Dynamic Systems $(D S)$ - technological objects in those the controlled processes of states modification occur. The general similarities of investigated DS of different application areas are a complex, nonlinear, multimodal objective function, the number of state points those could be used within a single study, large set of parameters and constraints of their simulation models. Such objective function's behavior requires solving global optimization tasks. Let us consider some examples of typical DSsimulation tasks for different application areas.

Aerodynamics. Until now it is necessary to carry out complicated, coupled simulations of air- and gas- flows distribution in complex aerodynamic objects those are frequently used for providing safety of working conditions. A typical example of such objects is a mine ventilation network. The main fragment of a mine ventilation system is an extraction area's ventilation system (Fig. 2).

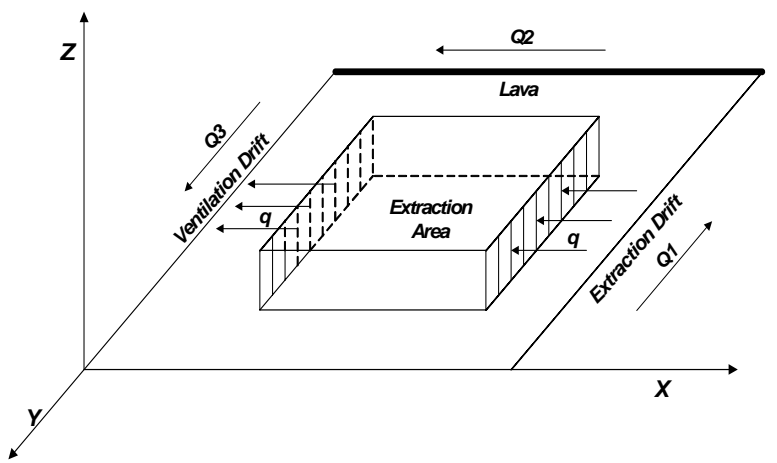

Fig. 2 - The structure of an extraction area's ventilation system.

The simulation model of an extraction area's ventilation system is formed by equations of an Extraction Drift

$$
\left\{\begin{array}{l}
-\frac{\partial p_{1}}{\partial \varkappa}=\frac{2 c}{F_{1}^{2}} Q_{1} \frac{\partial Q_{1}}{\partial \varkappa}+\frac{c}{F_{1}} \frac{\partial Q_{1}}{\partial t}+r_{1} Q_{1}^{2}+r_{1}(t) Q_{1}^{2} ; \\
-\frac{\partial p_{1}}{\partial t}=\frac{c a^{2}}{F_{1}} \frac{\partial Q_{1}}{\partial \varkappa}+\frac{c a^{2}}{F_{1}} g,
\end{array}\right.
$$

an approximated by parallel flows Extraction Area

$$
\left\{\begin{array}{l}
-\frac{\partial p_{g}}{\partial \varkappa}=\frac{c x}{k F_{g}} g+\frac{c}{m F_{g}} \frac{\partial g}{\partial t}+\frac{c}{l F_{g}{ }^{2}} g|g| ; \\
-\frac{\partial p_{g}}{\partial t}=\frac{c a^{2}}{m F_{g}} \frac{\partial g}{\partial \varkappa}
\end{array}\right.
$$

a Lava

$$
\left\{\begin{array}{l}
-\frac{\partial p_{2}}{\partial \varkappa}=r_{2} Q_{2}^{2}+\frac{c}{F_{2}} \frac{\partial Q_{2}}{\partial t} \\
-\frac{\partial p_{2}}{\partial t}=\frac{c a^{2}}{F_{2}} \frac{\partial Q_{2}}{\partial \varkappa}
\end{array}\right.
$$

a Ventilation Drift

$$
\left\{\begin{array}{l}
-\frac{\partial p_{3}}{\partial \varkappa}=\frac{2 c}{F_{3}^{2}} Q_{3} \frac{\partial Q_{3}}{\partial \varkappa}+\frac{c}{F_{3}} \frac{\partial Q_{3}}{\partial t}+r_{3} Q_{3}{ }^{2}+r_{3}^{\prime} Q_{3}{ }^{2} ; \\
-\frac{\partial p_{3}}{\partial t}=\frac{c a^{2}}{F_{3}} \frac{\partial Q_{3}}{\partial \varkappa}-\frac{c a^{2}}{F_{3}} g,
\end{array}\right.
$$

and boundary conditions [8].

Here $p_{1,2,3, g}$ - pressures; $Q_{1,2,3, g}$ - airflows; $\zeta-$ space coordinates, $\rho$ - air density; $a$ - speed of 
sound in air; $F_{1 . . g}$ - cross section's area; $r_{l . . g}-$ equivalent specific resistance; $r(t), r^{\prime}$ - variable aerodynamic resistances in drifts; $l_{l . . g}$ - length of an element; $v$ - cinematic viscosity index, $k$ penetration rate, $\mathrm{m}$ - rate voids.

Solving of referred to mine ventilation systems simulation tasks is the objective of scientific researches and developments of the Donetsk National Technical University (Ukraine) in cooperation with High Performance Computing Technology Center of Stuttgart (University of Stuttgart, Germany).

Molecular Dynamics. Classical Molecular Dynamics is used to simulate the dynamics of large systems, to gain a deeper understanding of the interactions between the atoms. It has an application in various scientific and engineering areas such as: chemical and process engineering, turbulent flows and process engineering, bio and enviromental processes and other. One example is the science test bed projects funded by Department of Earth Sciences of University of Cambridge [9] with the goal of primarily using grid-computing methods to facilitate simulations of environmental processes at a molecular level with increased levels of realism, achieved through being able to use larger samples, more complex systems, or through increased accuracy to better handle changes in chemical bonding.

\section{THE DISTRIBUTED SIMULATION ENVIRONMENT (DSE) FOR COMPLEX DYNAMIC SYSTEMS}

With the aim of virtual simulation models of different research areas unified development, their de-virtualization and decomposition into subsystems, distributed simulation service providing in scientific, industrial, business and educational researches [10] a Distributed Simulation Environment (DSE) is proposed (Fig. 3), which is a form of hardware, system software and simulation software resources system organization that supports all phases of models development, implementation and industrial use in order to requirements defined above. Hardware resources of DSE contain besides usual user work places also high-performance computer systems of SIMD- and MIMDArchitectures and clusters of nonparallel PCs. The Hardware of periphery systems is used for training simulators and their communication systems production, visualization, as well as for documentation of DSLB- and DSDP-simulation results. System Software of DSE consists of SIMDand MIMD-based parallel and distributed operation systems, software platforms of parallel and distributed programming, system means of periphery organization and functions providing.

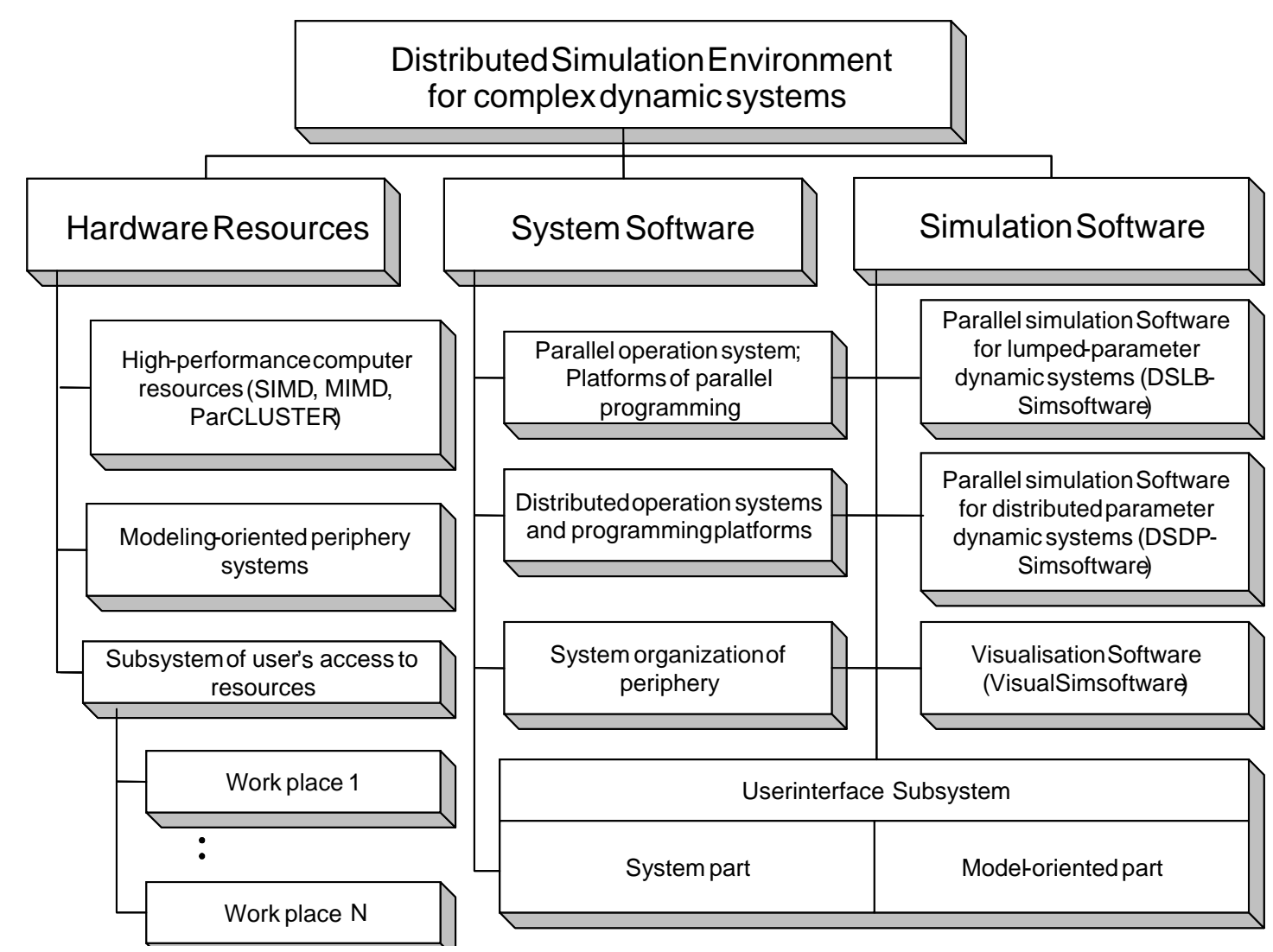

Fig. 3 - Structure organization of Distributed Simulation Environment for complex lumpedparameter and distributed parameter dynamic systems. 
Modeling and Simulation Software contains three main components - for DSLB- and DSDPsimulation and for providing visualization, those implement functionality of basic integrated in DSE simulation components which realize the unified algorithm of simulators development [11] topological analyzer (intended for preparing discreted primary topological information that is necessary for developing simulation models), equations generator (builds a set of equations systems on the basis of prepared by topological analyzer data and transforms them into virtual simulation models in conventional form) and equations solver (aims at cyclic realization of an algorithm of numerical solving generated equation systems in matrix-vector form, is developed separately for different numerical methods) [12].

With aim of systematization of the models development a DSE-decomposition into following subsystems is proposed [13]:

- user interface subsystem (consists of systemand modeling-oriented parts);

- subsystem of solving parallel equations;

- information transferring subsystem;

- processors load-balancing subsystem;

- data organization subsystem;

- subsystem of user access.

Developing of defined subsystems oriented on features of dynamic systems of different application areas like shown above results in implementation of technologically-oriented DSE those implement the accumulated experience of performing simulation tasks in such application areas on the principally new methodical and technological level.

\section{THE SYSTEM ORGANIZATION OF TECHNOLOGICALLY-ORIENTED DISTRIBUTED SIMULATION ENVIRONMENT FOR SOLVING INDUSTRIAL TASKS}

Organizationally a technologically-oriented DSE represents a software-technical infrastructure that provides users (engineers and operators of DSEapplication area, as well as researchers and simulators developers) with services those are foreseen within simulation service providing.

Within this infrastructure the following classes of DSE-resources can be defined (Fig. 4):

1. Workplaces of SSP-users (interconnected workplaces of specialists in predefined application area, computing centers, industrial automation systems).

2. Computing resources of problem domain's simulation tasks analysts (scientific and researching organizations - specialists in model-driven DS-supporting).

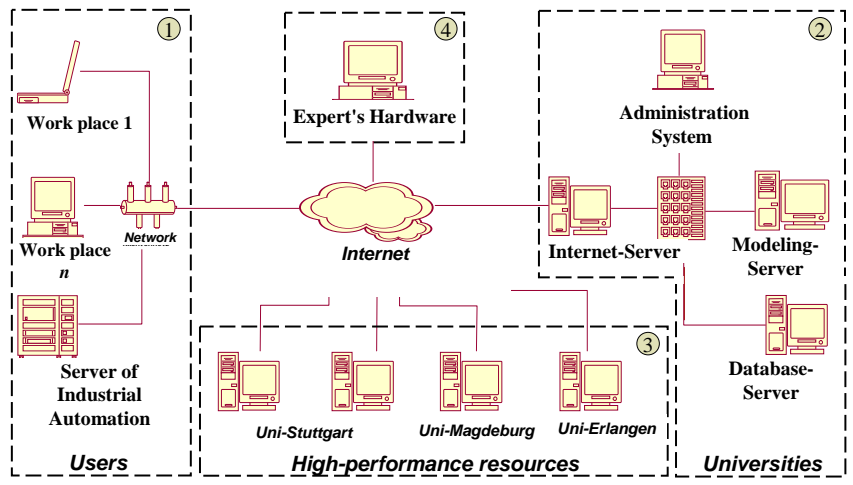

Fig. 4 - The organizational structure of a technologically-oriented distributed simulation environment.

3. Resource of HPC-owners - industrial and scientific organizations those are taking part in simulation researches supporting by their high-performance computer environments.

4. Resources of decision-making's supporting systems of experts and controlling organizations those specialize in predefined application area.

This resource sharing infrastructure might aim at the achieving the highest possible performance in solving widespread industrial simulation tasks.

The system organization of technologicallyoriented DSE includes 3 basic layers of hardware and software resources (Fig. 5):

- $\quad$ Users layer

- $\quad$ Server layer

- $\quad$ Supercomputer layer

This SimGrid-system organization foresees easy and transparent accessing heterogenous user resources (e.g. PCs, laptops, mobile devices etc.) to DSE-simulation services providing uniform highperformance computational facilities. Models management, simulation jobs remote submission and monitoring, Grid-resource reservation, secure data transport and another user tasks performe through the web-interface which is specially developed for different application areas and hierarchical types of users within each specified application area.

The main administrative domain of DSE is the SimGrid-Server, the main functional components of that (models server, internet server, database server) service the high-level functionality of SimGridSystem - users authorization and authetification, simulators management, provide user access to simulation services. The simulators are developed as Grid-Services according to OGSA-standard [14] with further distribution of simulators set within high performance computing environment of supercomputer layer, the resources of which belong to different administrative domains, e.g., different research institutions in different countries or different companies. 


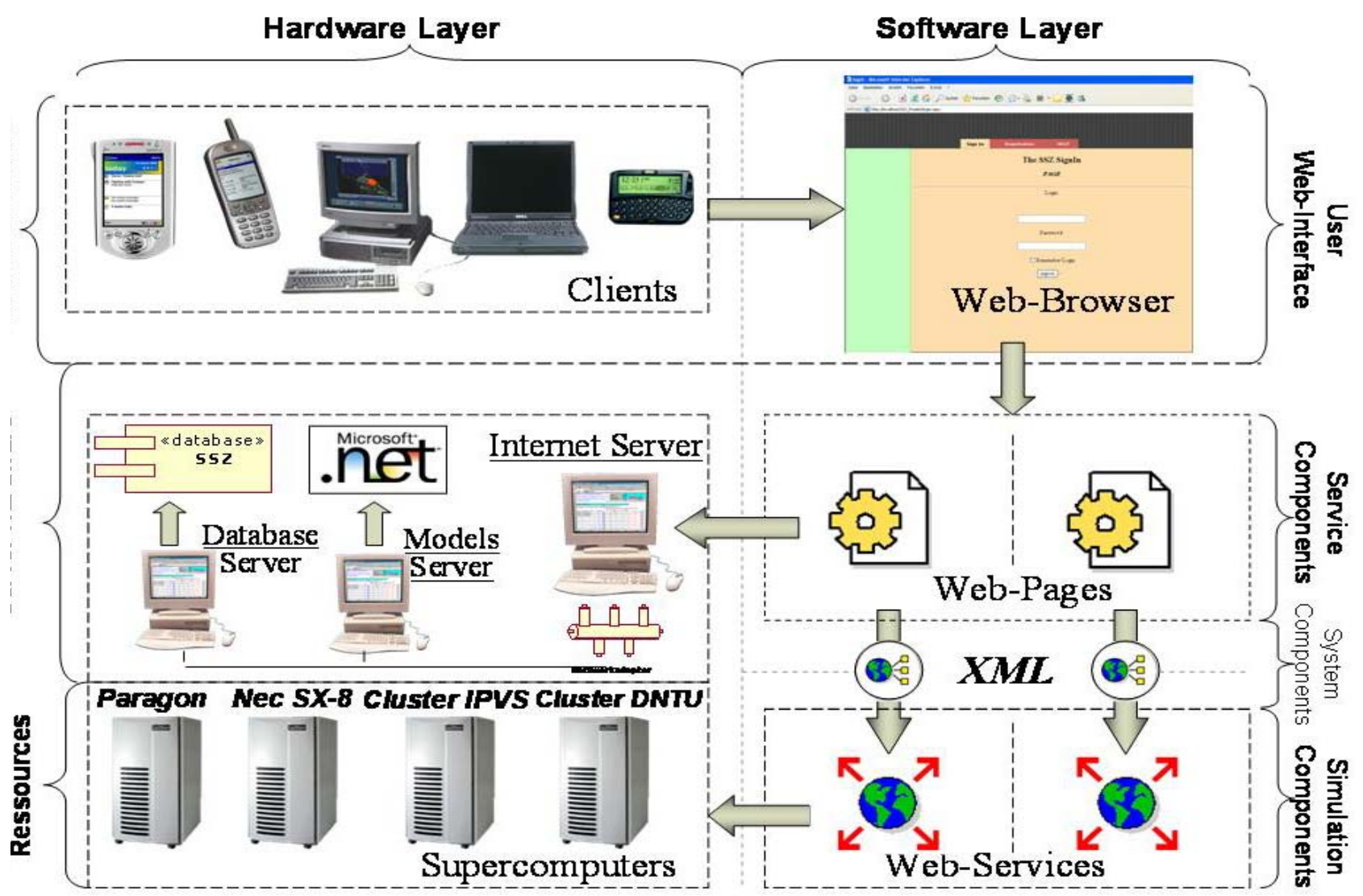

Fig. 5 - The system organization of Grid-based distributed simulation environment.

\section{SIMULATION SERVICES DEVELOPMENT IN DSE-DESCRETE EVENT SIMULATION FRAMEWORK}

Developing and providing simulators as application specific services requires a discrete event simulation framework that meets the defined for DSE requirements and provides necessary level of quality-of-service as well. This framework is tightly coupled with developments of DSE-components, what causes their orientation not only on simulation service but also on development tasks. With this aim special problem-oriented Simulation and Servicing Centers (SSC) could be proposed [15] that integrate DSE for specified application area, as well as software technologies and platforms of their development. Furthermore, development of SSC aims at practical providing defined subsystems of technologically-oriented DSE for scientific and industrial on-line simulation in suitable for experts of specified application area user-friendly form.

In this context an especial role obtains using progressive informational technologies that aim at organization and supporting of software components development that realize simulators functionality according to principals of DSE system organization.

\subsection{Using Unified Modeling Language (UML) for the Development of Software Components of DSE-Simulators}

The complexity of DSE-components and function algorithms of their implementation within defined SSC's functionality, the distributed nature of technologically-oriented DSE's developers leads to a necessity of using a special technology of component-based software systems development. In connection to this an especial interest awakes choosing a special notation of SSC-projects definition, modeling components development's management, supporting and documentation as well. As a possible unified solution for all tasks of simulators development and further supporting, system integration within another SSC's service and modeling components could be proposed UMLbased technologies. UML [16] is a wide-spread modeling language for software and systems development which became last years practically a standard of object-oriented simulation. The UML can be effectively used for solving following DSE's development tasks: logical notion of researched objects, component-based simulators development, using simulators for solving industrial tasks, DSE's system organization, simulation study's procedure. 


\subsection{The Unified Process of Simulation Services Development}

With the aim of simulation services development a based on integration of Rational Unified Process [17] with Waterfall Model, tightly coupled with UML technologically-oriented, Use-Case-Driven, iterative and architecture centric unified process is proposed (Fig. 6) that includes the following development phases: requirement managing, system analysis, projecting, realization. Each development phase is performed by means of defined set of UMLdiagrams development. Herewith the actual task was adapting defined phases to peculiarities of SSP in the most common application areas of simulation technology (e.g. coal industry, hydraulic, electrical engineering) and to the features of proper to them dynamic systems formal description which contains two basic parts - mathematical description and topological description. The adoption was performed through the developing a new diagrams set that is not foreseen within UML 2.0 standard [16].

Based on experiences for real industrial projects [18], the following diagrams were proposed: for the requirements managing: the simulators diagram (aims at unified representing simulation models of continuous DS-processes, their ordering within application area's models hierarchy [18], defining topological and functional relationships between models as well), the application area diagram (contains a UML-notated description of researched DS, as well as their properties and features); for the system analysis: the advanced activity diagram (represents messages interchange and informational coupling among modelling and service DSEsoftware components through the special classification symbol), the timing diagram (used for specification of researched DS's behaviour in time, documentation of time parameters of simulation study proceeding as well); for the projecting: the advanced deployment diagram (is provided by UML-notion of coupling simulation services and their components with groups of distributed DSE's resources); for the realization: the advanced class diagram (represents object-oriented organizational structure of modelling components realization), the sequence diagram (contains DSE's resources as participants and defines use of simulation services by solving the simulation tasks), the data diagram (aims at representing the structure of informational layer of SimGrid infrastructure).

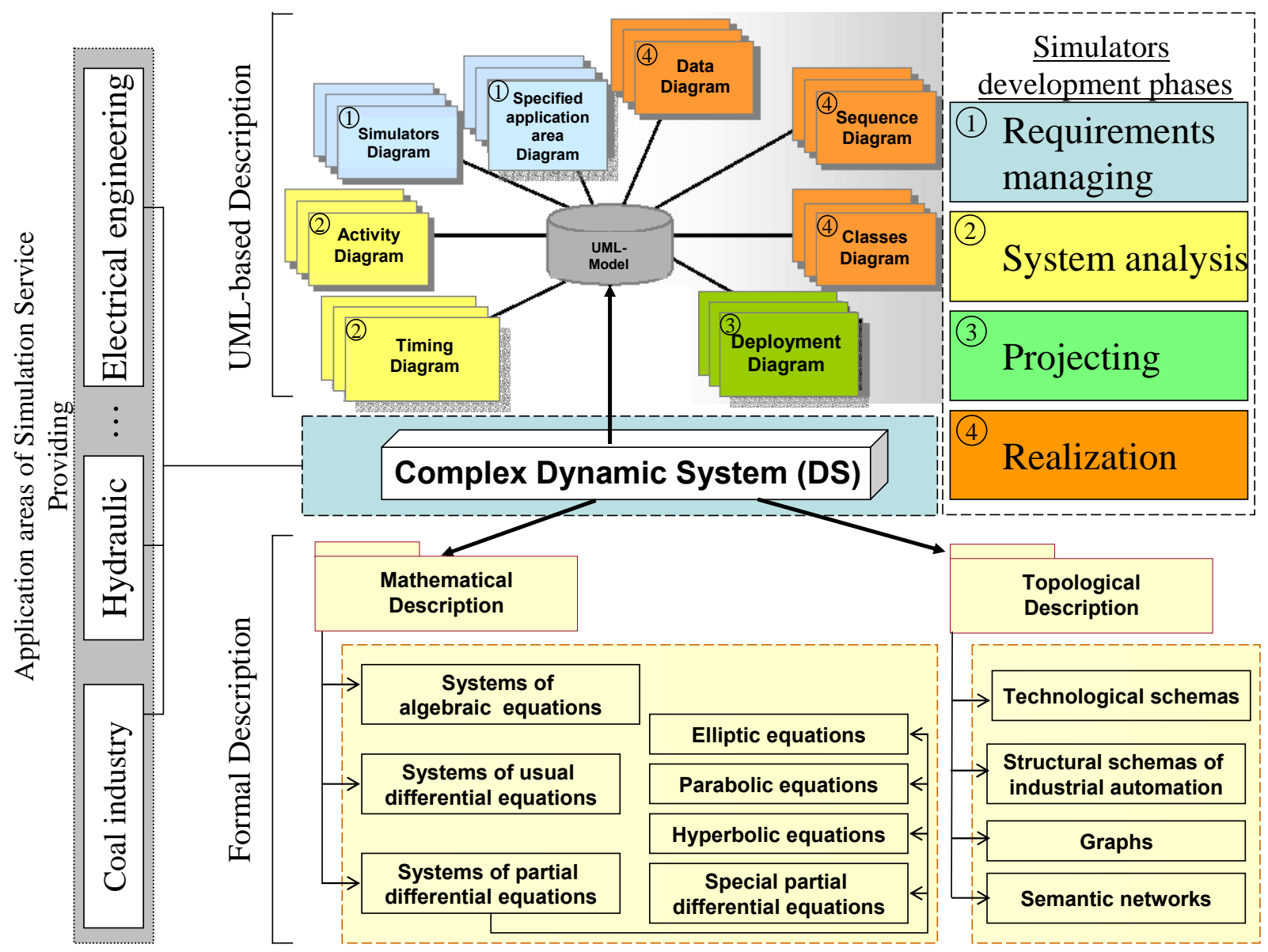

Fig. 6 - The unified process of simulation services development for complex dynamic systems. 


\section{CONCLUSION}

This article gave a short introduction into the motivation and the current implementation of Grid infrastructures for solving tasks of industrial simulation. Although research groups all over the world are working towards development of Gridenvironments that would be available to groups of simulation scientists independent of their local environment of computers, programs, data and information resources, considerable additional research and development for based on Grid technologies Simulation Service Providing in different application areas is required. As result of this a widespread adoption by scientific organizations and research groups and a broader use of Grid technologies for solving industrial tasks are expected. This requires complex approaches development, one of them - a technologicallyoriented distributed simulation environment, as well as system organization of simulation services providing an easy access to Grid infrastructure of simulators developers and engineering stuff as well is proposed in this article. The future developments will concentrate on further development of simulation models and services for dynamic systems as well as their implementation for solving industrial tasks as components of problem-oriented Simulation and Servicing Centres for specified application areas.

\section{REFERENCES}

[1] P. Fishwick. Web-Based Simulation: Some Personal Observations. Proceedings of the 1996 Winter Simulation Conference (1996). pp. 772-779.

[2] P.Fishwick. Using XML for Simulation Modeling. Proceedings of the 2002 Winter Simulation Conference (2002).

[3] J. Kuljis, R. Paul. A review of web based simulation: whiter we wander? Proceedings of the 2000 Winter Simulation Conference, Orlando Florida (2000). pp. 1872-1881.

[4] T. Wiedemann. Simulation Application Service Providing (SIM-ASP). Proceedings of the 2001 Winter Simulation Conference (2001).

[5] T. Wiedemann. Next Generation Simulation environments Founded On Open Source Software and XML-Based Standard Interfaces. Proceedings of the 2002 Winter Simulation Conference (2002).

[6] F. Bry, W. Nagel, M. Schroeder. GridComputing. Informatik Spektrum 13 (2004). pp. 552-554.

[7] F. Berman, A. Hey, G. Fox. Grid computing: Making the global infrastructure a reality.
Wiley (2003).

[8] O. Cheptsov, V. Svjatnyj, R. Hohmann. Die Entwicklung eines Simulations- und servicezentrums für gegebenes Gegenstandsgebiet. Collected Volume of Scientific Papers of Donetsk National Technical University. SCAD-2005. Volume 93 (2005). pp. 151-158.

[9] M. T. Dove and N.H. De Leeuw. Grid computing and molecular simulations: the vision of the eMinerals project. Molecular Simulation, Vol. 31, No. 5, (2005). pp. 297301.

[10] V. Svjatnyj., A. Masjuk, O. Smagin, H.-J. Bungartz. Interaktive Modellierung, Simulation und Prozessführung in einer parallelen problemorientierten Simulationsumgebung. F.Hülsemann u.a. (Hrsg.), Tagungsband 18. ASIM-Symposium Simulationstechnik, Erlangen (2005). pp. 742747.

[11] A. Cheptsov. The UML-Approach Of Developing Discrete Simulators (On Instance Of Coal Industry's Objects). Simulation and Computer Graphic, Donetsk (2008). pp. 354358.

[12] L.P. Feldmann, V.A. Svjatnyj, V.V. Lapko, E.-D. Gilles, M. Zeitz, A. Reuter, K. Rothermel. Parallel Simulation Technology. Problems of Simulation and Computer Aided Design of Dynamic systems. Collected Volume of Scientific Papers of Donetsk National Technical University. SCAD-1999 (1999). pp. 9-19.

[13] V. Svjatnyj, K. Teplinskiy, V. Trubarov. Optimisation Problems in the TechnologicalOriented Parallel Simulation Environment. F.Hülsemann u.a. (Hrsg.), Tagungsband 18. ASIM-Symposium Simulationstechnik, Erlangen (2005). pp.582-587.

[14] M. Gyimesi, F. Breitenecker. Simulation Service Providing als Web Service. F.Hülsemann u.a. (Hrsg.), Tagungsband 18. ASIM-Symposium Simulationstechnik, Erlangen (2005).

[15] O. Cheptsov, V. Svjatnyj, O. Beljaev, V. Lapko, O. Schkrebez Zur Entwicklungsorganisation des Simulations- und Servicezentrums für die Kohleindustrie. Simulationstechnik 18. Symposium in Erlangen, September (2005). pp.554-559.

[16] R. Miles, K. Hamilton. Learning UML 2.0. O'Reilly (2006). 269 p.

[17] Sommerwille Ian. Software Engineering. 6.Auflage. Addison Wesley Verlag (2001).

[18] O. Cheptsov. The system organization and basic algorithms of the Simulation- and 
Servicecentre for the coal industry. IEEE Proceedings International Conference "Modern Problems of Radio Engineering, Telecommunications and Computer Science TCSET'2006”" (2006), pp. 205-207.

$\begin{gathered}\text { Dr.-Ing. Alexey Cheptsov } \\ \text { holds a diploma (M.Sc.) of } \\ \text { computer systems and } \\ \text { networks and a diploma of } \\ \text { economics (Dipl.-Fin.) of the } \\ \text { Donetsk National Technical } \\ \text { University (Ukraine). He has } \\ \text { done a Ph.D. in automated } \\ \text { control systems and }\end{gathered}$
progressive informational technologies at the
G.Pukhov's Institute of Simulation Problems in
Power Engineering, National Academy of Sciences
of Ukraine.
At present he works as an Assistant Professor of the
Department of Computer Science of the Donetsk
National Technical University. He is the leader of
Distributed and Parallel Computing Group. His main
research interests are Grid technology, high-
performance computation, embedded systems,
automotive electronic engineering.

